\title{
Vulnerabilidad y riesgo sísmico de los edificios residenciales estudiados dentro del Plan Especial de evaluación del riesgo sísmico en la Comunidad Valenciana
}

\author{
Vulnerability and seismic risk of the residential buildings in the Special Plan \\ against seismic risk of Valencia region
}

$\underline{\text { B. Serrano-Lanzarote }}^{(*)}$, R. Temes-Córdovez ${ }^{(*)}$

\section{RESUMEN}

Se presenta un estudio sobre la vulnerabilidad sísmica de edificios residenciales en la Comunidad Valenciana, como parte del Plan Especial frente al riesgo sísmico que dicha comunidad tiene obligación de elaborar al tener poblaciones con intensidades sísmicas superiores a VII grados, según la Escala Macrosísmica Europea. Se exponen los resultados y metodología seguida para identificar las características constructivas de la región, antigüedad de sus edificios, asignar clases de vulnerabilidad sísmica, estimar daños potenciales ocasionados por un probable sismo, así como cartografiar el riesgo derivado. Se destaca la estimación de un daño grave del $13 \%$ del número de edificios para la provincia de Alicante, disminuyendo en las provincias de Valencia y Castellón hasta el $8 \%$ y el o,2 \%, respectivamente. Para la gestión de la información se ha creado una geodatabase, administrada por un Sistema de Información Geográfica, herramienta que ha resultado muy eficaz, especialmente para la respuesta inmediata a preguntas multicriterio.

Palabras clave: Vulnerabilidad; sismo; riesgo; SIG; Comunidad Valenciana.

\section{ABSTRACT}

This paper presents a study about the seismic vulnerability of residential buildings in the Valencian region, as part of the Special Plan for the seismic risk. The regional government has the obligation to develop these type of plans in areas with seismic intensities higher than VII degrees, accord to the European Macroseismic Scale. The methodology used to identify the constructive characteristics in the region, age of buildings, to assign seismic vulnerability classes, to estimate potential damage caused by a probable earthquake and mapping the risk arising, is presented. We highlighted the estimation of an average high damage of $13 \%$ of the number of buildings in the Alicante region, decreasing in Valencia and Castellon to $8 \%$ and $0.2 \%$, respectively. To manage data a geodatabase has been created, managed by a Geographic Information System, a very effective tool, specially to the immediate response to multicriteria questions.

Keywords: Vulnerability; earthquake; risk; GIS; Comunidad Valenciana.

(*) Universidad Politécnica de Valencia (España).

Persona de contacto/Corresponding author: apserlan@mes.upv.es (B. Serrano-Lanzarote).

Cómo citar este artículo/Citation: Serrano-Lanzarote, B., Temes-Córdovez, R. (2015). Vulnerabilidad y riesgo sísmico de los edificios residenciales estudiados dentro del Plan Especial de evaluación del riesgo sísmico en la Comunidad Valenciana. Informes de la Construcción, 67(539): e104, doi: http://dx.doi.org/10.3989/ic.13.182.

Licencia / License: Salvo indicación contraria, todos los contenidos de la edición electrónica de Informes de la Construcción se distribuyen bajo una licencia de uso y distribución Creative Commons Reconocimiento no Comercial 3.o. España (cc-by-nc). 


\section{INTRODUCCIÓN}

Ante el riesgo sísmico, la directriz básica de planificación de Protección Civil indica los requisitos mínimos que se deben cumplir para la elaboración de los correspondientes «Planes Especiales de Protección Civil» y establece que dichos planes se deben elaborar en aquellas comunidades autónomas donde, en el mapa de peligrosidad sísmica de España, incluido en la Norma de construcción sismorresistente (1), se prevea una intensidad sísmica igual o superior a VII grados para un periodo de retorno de 500 años. Según la Escala Macrosísmica Europea, EMS-98 (2) a partir de una intensidad VII se comienzan a producir daños importantes en las estructuras.

La Comunidad Valenciana se encuentra cercana a la zona de contacto entre las grandes placas Euroasiática y Africana, lo que favorece la ocurrencia periódica de actividad sísmica, cuya intensidad se puede calificar en general de moderada, pero de relativa importancia en la Península Ibérica. La mayor peligrosidad sísmica de la comunidad se concentra en la comarca del Bajo Segura, principalmente al sur de la provincia de Valencia y toda la de Alicante.

En épocas recientes determinadas zonas de la misma se han visto afectadas por terremotos de considerable magnitud, como el terremoto de Torrevieja que, el 21 de marzo de1829, causó 389 muertos, 375 heridos y destruyó más de 5.000 viviendas y que representa el suceso sísmico más destructivo acaecido en la región.

Recientes estudios desarrollados sobre peligrosidad en la Comunidad Valenciana por la Universidad de Alicante (3), contemplan la probabilidad de ocurrencia de movimientos sísmicos con una intensidad igual o superior a VII, según la EMS-98 (2), para un período de retorno de 500 años, información que queda reflejada en el mapa de la Figura 1.

Para obtener más información sobre datos y mapas de peligrosidad en la Comunidad Valenciana, se aconseja la consulta del documento elaborado por la Universidad de Alicante y dirigido por el profesor Giner Caturla (3).

Estos antecedentes justifican el desarrollo de estudios que permitan la elaboración de los correspondientes mapas de riesgo sísmico que deberán proporcionar una visión lo más precisa posible acerca de las probables consecuencias de una catástrofe sísmica en el territorio, lo que permitirá hacer previsiones sobre los medios y recursos necesarios para la intervención y localizar la infraestructura de apoyo utilizable para el auxilio del área afectada.

Conforme a los antecedentes expuestos, la Consejería de Gobernación, organismo responsable de las competencias de Protección Civil de la Generalidad Valenciana, ha desarrollado el Plan Especial frente al Riesgo Sísmico de la Comunidad Valenciana (4) para lo que ha sido imprescindible elaborar los correspondientes estudios técnicos, relativos tanto a peligrosidad como a vulnerabilidad sísmica, en función de los distintos niveles de intensidad de un posible sismo. Sandi (5) define la peligrosidad y la vulnerabilidad sísmicas de la siguiente manera:

- La peligrosidad sísmica representa la probabilidad de ocurrencia, dentro de un período específico de tiempo y dentro de un área dada, de un movimiento sísmico del terreno de un nivel de severidad determinado.
Giner Caturla (3) la define como la probabilidad de que el valor de un parámetro que cuantifica el movimiento del suelo, sea superado en un periodo de tiempo, también llamado periodo de retorno. Por ejemplo, un área con una peligrosidad sísmica de grado de intensidad VII, para un periodo de retorno de 500 años, implica que existe una probabilidad del $10 \%$ de que se produzca una sacudida del suelo de grado VII, en un periodo de retorno de 50 años. También podría definirse como la probabilidad anual, de que ocurra una sacudida del suelo de grado VII, del 1/500, es decir del o,2 \%.

- La vulnerabilidad sísmica se define como la predisposición intrínseca de una estructura, grupo de estructuras o de una zona urbana completa de sufrir daño ante la ocurrencia de un movimiento sísmico de una severidad determinada.

En consecuencia, la vulnerabilidad está directamente relacionada con las características de diseño de la estructura. Para poder estimarla se deben efectuar estudios que comprendan las construcciones cuya destrucción, con probabilidad razonable, pueda ocasionar víctimas, interrumpir un servicio imprescindible para la comunidad o aumentar los daños por efectos catastróficos asociados. Una elevada

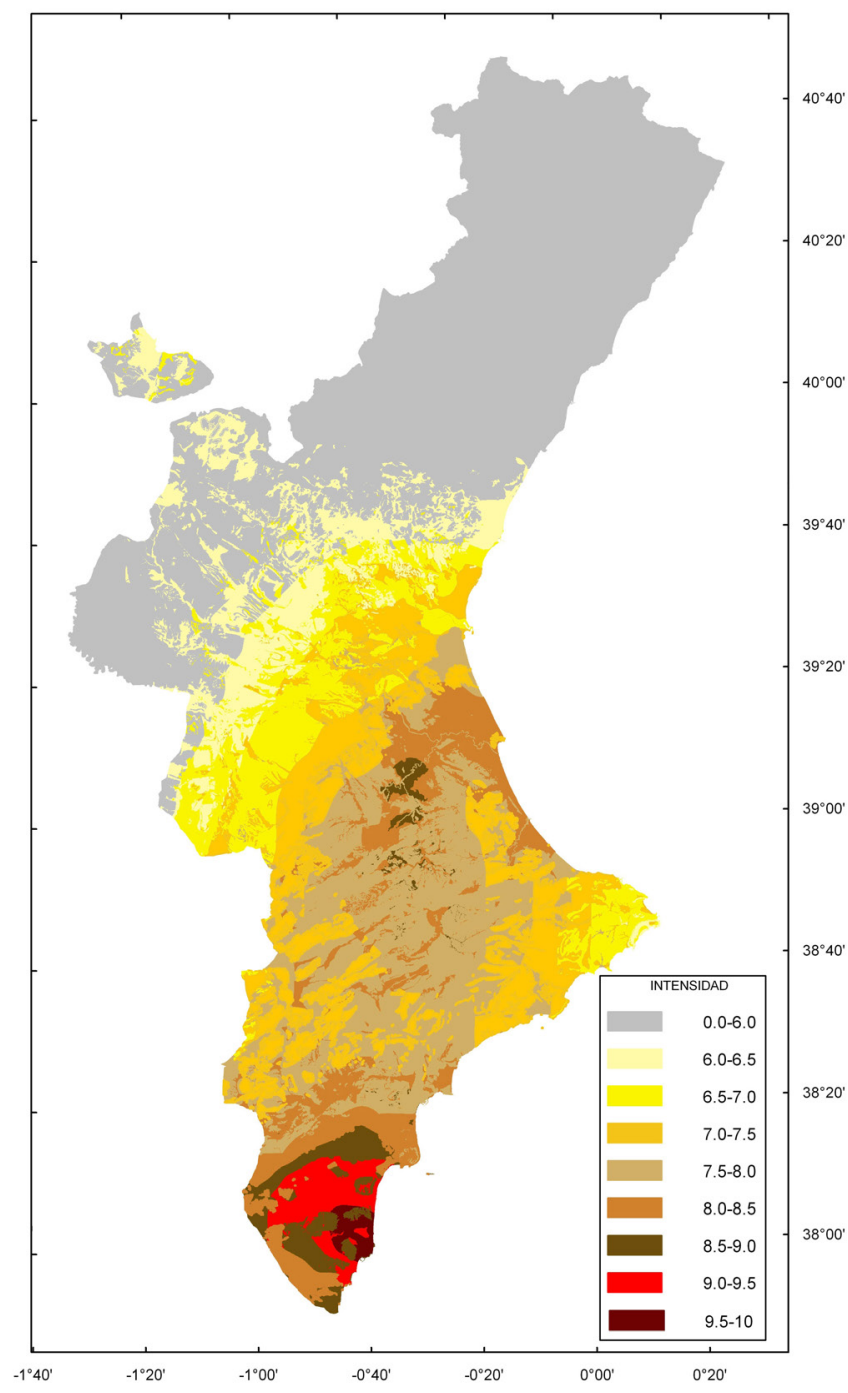

Figura 1. Mapa de intensidad sísmica esperada en la Comunidad Valenciana para un periodo de retorno de 500 año (3). 
vulnerabilidad puede ocasionar importantes daños, a pesar de producirse en un área con una peligrosidad sísmica moderada.

Según Perepérez (6) el riesgo es función de la peligrosidad, de la vulnerabilidad y de los elementos amenazados y representa los daños a la población, pérdidas materiales y paralización de la actividad económica [1].

$$
\mathrm{R}=\mathrm{R}(\mathrm{H}, \mathrm{V}, \mathrm{E})
$$

El objeto del presente artículo es presentar el estudio de vulnerabilidad sísmica de la Comunidad Valenciana elaborado por los autores. Para generar esa información, se ha recurrido a una metodología simplificada ya empleada en otras regiones de España (7), mediante la cual se asignan clases de vulnerabilidad en función de la edad de la edificación. La novedad de este estudio es que se ha generado una clasificación de los tipos edificatorios más representativos en la Comunidad Valenciana, y para cada uno de ellos se ha propuesto una distribución, según los periodos constructivos considerados, para finalmente asignarles una clase vulnerabilidad. Dicha información combinada con los datos sobre peligrosidad de cada población (3), ha permitido estimar los daños potenciales ocasionados por un probable sismo y cartografiar el riesgo derivado para todos los municipios de la comunidad. Aunque no es objeto de este artículo, el estudio elaborado incluye información sobre el número de posibles víctimas, los daños en la infraestructura, los grados de afectación de instalaciones y servicios imprescindibles para la atención de la emergencia, así como posibles daños en instalaciones capaces de dar lugar a peligros asociados.

Desde el punto de vista instrumental señalamos como aportación principal la gestión de los datos alfanuméricos y gráficos del análisis mediante una geodatabase ${ }^{1}$ administrada a través de un Sistema de Información Geográfica (SIG). El uso de los SIG para gestionar los datos en este tipo de Planes Especiales no supone una novedad, pero sí se presenta como aportación las ventajas que ha demostrado el uso de las geodatabases, frente al almacenamiento tradicional de la información en bases de datos relacionales simples.

\section{PROCEDIMIENTO PARA LA ASIGNACIÓN DE CLASE DE VULNERABILIDAD A LOS EDIFICIOS DE LA COMUNIDAD VALENCIANA}

Para estimar la vulnerabilidad en los edificios se ha seguido un método simplificado basado en las clases de vulnerabilidad. El procedimiento se desglosa de la siguiente manera:
1) Estudio de fuentes básicas de información cuantitativa $y$ cualitativa del parque edificado en la Comunidad Valenciana. Principalmente se ha trabajado con el Censo de Población y Vivienda 2001 elaborado por el Instituto Nacional Estadístico (INE) ${ }^{2}$ y con datos sobre inspecciones de edificios construidos entre los años 1950 y $1980^{3}$ en la Comunidad Valenciana (8).

2) Análisis de las características constructivas y de diseño de los edificios de la Comunidad Valenciana en diferentes periodos y definición de tipos estructurales básicos fundamentados en diversos estudios (9) y (10).

3) Asignación de vulnerabilidad adecuada a la Escala Macrosísmica Europea, EMS-98 (2), a cada uno de los edificios y tipos estructurales identificados.

4) Ponderación de cada clase de vulnerabilidad por período constructivo mediante el desarrollo de matrices de asignación de vulnerabilidad.

Gestión de resultados y salida gráfica de cartografía a través de una geodatabase administrada mediante un SIG.

\subsection{Fuentes de información}

Para asignar vulnerabilidad a los edificios es necesario disponer de información que permita identificar su sistema estructural. Atendiendo a la amplitud del ámbito de estudio, se ha recurrido a métodos indirectos de análisis basados en el uso de información estadística sistematizada para identificar los tipos estructurales de los edificios, en función de la edad, la calidad constructiva, el estado de conservación y las alturas.

Como fuentes de información se ha trabajado con el Censo de Población y Vivienda 2001 que dispone de información detallada para los edificios con uso destinado principalmente a vivienda. Otra fuente de datos ha sido el estudio elaborado por el Instituto Valenciano de la Edificación (IVE) en el que se recoge información relativa a las inspecciones técnicas llevadas a cabo en la Comunidad Valenciana entre 1992 y 2008 (8).

En la Comunidad Valenciana no se cuenta con datos directos relativos al comportamiento de la edificación frente al sismo, al no acontecer en los últimos años sismos suficientemente significativos. Por ello se han empleado fuentes de información de áreas de características similares como es la Región de Murcia ${ }^{4}$.

\footnotetext{
${ }^{1}$ Las geodatabases se definen como una colección de datasets geográficos de varios tipos. Se denominan dataset a un conjunto de datos organizados normalmente en formato de tabla y vinculados con un conjunto determinado de variables.

2 Procede justificar el uso de una fuente de información anterior al año 2001 como es el caso del Censo de Población y Viviendas 2001. Se trata de la única base de datos sistemática de información sobre edificios que abarca todo el territorio nacional y que además tiene garantizada su renovación cada 10 años. Los cambios producidos en la última década si bien han sido significativos, contabilizan las nuevas viviendas que no comprometen la estructura interna de distribución cronológica del parque edificado, aspecto éste fundamental en el uso de dicho censo. http://www.indec.gov.ar/webcenso/index.asp.

3 Se justifica el uso de este intervalo por ser el período en el que se recogen las décadas de mayor intensidad en la construcción en la Comunidad Valenciana coincidiendo además con el intervalo en el que es posible detectar peores condiciones constructivas en la edificación. Además el intervalo 1950-80 es representativo del $30 \%$ del parque edificado en la Comunidad Valenciana.

4 El último sismo relevante ocurrido en la Región de Murcia tuvieron lugar en Lorca, el año 2011. Los resultados y conclusiones de los diversos estudios elaboraos al respecto, no han podido considerarse en el trabajo expuesto en el presente artículo, debido a que se finalizó en octubre del año 2010 .
} 


\subsection{Tipos estructurales definidos}

En general, la forma más extendida de clasificar la vulnerabilidad de la edificación en Europa es la basada en escalas macrosísmicas. La más comúnmente utilizada es la Escala Macrosísmica Europea, EMS-98 (2), que considera seis clases de vulnerabilidad, según el tipo de estructura del edificio, distinguiendo entre estructuras de fábricas y de hormigón armado.

Este trabajo ha propuesto por vez primera en la Comunidad Valenciana, una correspondencia entre la vulnerabilidad y la edificación, basándose para ello en la valoración de un conjunto de variables como son el reconocimiento de los tipos estructurales, de la antigüedad, de su ubicación geográfica y de otras características significativas como el nivel de diseño sismorresistente de los edificios en función de la existencia o no de normativa de diseño sísmico en España. Hay que indicar que se han recogido los casos considerados como representativos, por darse con mayor frecuencia en el parque de viviendas construido, aunque pueden encontrarse tipos de edificios con unas características particulares no contemplados en esta aproximación más generalista. La Tabla 1 recoge el esquema clasificatorio básico.

Esta asignación de vulnerabilidad a cada tipo, se plantea inicialmente considerando la edad de construcción del edificio, para posteriormente ajustarla y matizarla con un análisis más profundo en función de un criterio razonable de base experta, establecido mediante la consulta a un grupo de especialistas en la materia con el fin de crear convergencia de opiniones. Con dicho criterio se tienen en cuenta factores como las características constructivas y de disposición estructural, el nivel de calidad de los materiales y la ejecución, las condiciones de rigidez de los forjados, el nivel de aplicación de las condiciones constructivas, el confinamiento y el nivel de diseño sismorresistente, así como consideración de casos críticos como plantas débiles y pilares cortos. Además del procedimiento anterior, se consideró necesario contrastar dicha información mediante la aplicación del método del índice de vulnerabilidad, también conocido como Método Italiano (11), en una selección de edificios existentes y representativos de cada una de los tipos estructurales propuestos. Dicho método se ha aplicado con buenos resultados en los terremotos de Almería en 1993-94 y Murcia en 1999, por Yépez (12) y Mena (13), respectivamente.
El método evalúa una serie de parámetros que permiten cuantificar la predisposición de un edificio a sufrir daños por un evento sísmico. Considera aspectos como la organización del sistema resistente, la configuración en planta y en elevación, los cimientos, el tipo de cubierta, el estado de conservación, etc.

Dichos parámetros se evalúan con unas clases (A, B, C o D), para asignarles un valor numérico $\left(K_{i}\right)$, que varía entre o y 45. Este valor es ponderado por un coeficiente de peso $\left(W_{i}\right)$, que varía entre 0,25 y 1,50, para indicar la importancia que tiene cada uno de ellos en la asignación final del índice de vulnerabilidad. La Tabla 2 recoge los parámetros y valores para edificios con estructura de mampostería no reforzada.

El índice de vulnerabilidad $I_{v}$, queda definido por la expresión [2], que representa la suma ponderada de cada uno de los valores de cada parámetro evaluado:

$$
I_{V}=\sum_{i=1}^{11} K_{i} \cdot W_{i}
$$

Siendo,

$I_{v}$, es el índice de vulnerabilidad

$K_{i}$, representa cada uno de los parámetros del edificio evaluados, variando el subíndice $i$ desde 1 hasta 11

$W_{i}$, peso asignado a cada parámetro $K_{i}$

$\mathrm{Al}$ analizar la ecuación se puede deducir que el índice de vulnerabilidad define una escala continua de valores desde o hasta 382,5. La ecuación puede normalizarse para obtener índices variando entre o y 100 o entre o y 1 , que corresponderían a un edificio nada vulnerable o muy vulnerable, respectivamente.

A modo de ejemplo, en el caso de las estructuras de mampostería clasificadas como tipo F1 se obtuvo un índice de 0,75 y para el F2 los valores oscilaban entre 0,25 y 0,55. No se llegaron a obtener índices por debajo de 0,15 , a los que el método asigna una vulnerabilidad baja, por lo que en ningún caso se les pudo asignar vulnerabilidades de clase C o D.

Tabla 1. Clasificación de tipos estructurales de edificios residenciales en la Comunidad Valenciana.

\begin{tabular}{|l|c|l|c|}
\hline \multirow{2}{*}{ Material } & Código tipo & \multicolumn{1}{|c|}{ Descripción } & $\begin{array}{c}\text { Clase de } \\
\text { vulnerabilidad }\end{array}$ \\
\hline \multirow{2}{*}{ Fábrica } & F1 & Estructura de muros de carga de piedra en seco y tapial & A \\
\cline { 2 - 5 } & F2 & Estructura de muros de carga de mampostería o de fábrica de ladrillo < 5 plantas & A / B \\
\hline \multirow{4}{*}{ Mixta } & X1 & $\begin{array}{l}\text { Estructura mixta de mampostería o de fábrica de ladrillo y pórticos de fábrica de } \\
\text { ladrillo < 1950 }\end{array}$ & A / B \\
\cline { 2 - 5 } & X2 & $\begin{array}{l}\text { Estructura mixta de mampostería o de fábrica de ladrillo y pórticos de hormigón } \\
\text { armado 1950-1970 }\end{array}$ & B \\
\hline \multirow{3}{*}{$\begin{array}{l}\text { Hormigón } \\
\text { armado }\end{array}$} & H1 & Estructura porticada de hormigón armado, 1930-1969 & B \\
\cline { 2 - 5 } & H2 & Estructura porticada de hormigón armado, 1970-1995 & B / C \\
\hline \multirow{2}{*}{ Acero } & A1 & Estructura porticada de hormigón armado, >1995 & C D / E \\
\cline { 2 - 5 } & A2 & Estructura porticada de nudos semirígidos & C / D \\
\cline { 2 - 5 } & A3 & Naves industriales & C / D \\
\hline
\end{tabular}


Tabla 2. Valores numéricos de cada parámetro del índice

de vulnerabilidad para edificios con estructura de mampostería no reforzada.

\begin{tabular}{|r|l|c|c|c|c|c|}
\hline \multirow{2}{*}{$\mathbf{i}$} & \multirow{2}{*}{ Parámetro } & \multicolumn{3}{c|}{ Valor de Ki, según clase } & \multirow{2}{*}{ Wi } \\
\cline { 3 - 6 } & & $\mathbf{A}$ & $\mathbf{B}$ & $\mathbf{C}$ & $\mathbf{D}$ & \\
\hline $\mathbf{1}$ & Organización del sistema resistente & 0 & 5 & 20 & 45 & 1,00 \\
\hline $\mathbf{2}$ & Calidad del sistema resistente & 0 & 5 & 25 & 45 & 0,25 \\
\hline $\mathbf{3}$ & Resistencia convencional & 0 & 5 & 25 & 45 & 1,50 \\
\hline $\mathbf{4}$ & Posición del edificio y disposición & 0 & 5 & 25 & 45 & 0,75 \\
\hline $\mathbf{5}$ & Diagramas horizontales & 0 & 5 & 15 & 45 & 1,00 \\
\hline $\mathbf{6}$ & Configuración en planta & 0 & 5 & 25 & 45 & 0,50 \\
\hline 7 & Configuración en elevación & 0 & 5 & 25 & 45 & 1,00 \\
\hline $\mathbf{8}$ & Separación máxima entre muros & 0 & 5 & 25 & 45 & 0,25 \\
\hline $\mathbf{9}$ & Tipo de cubierta & 0 & 15 & 25 & 45 & 1,00 \\
\hline $\mathbf{1 0}$ & Elementos no estructurales & 0 & 0 & 25 & 45 & 0,25 \\
\hline $\mathbf{1 1}$ & Estado de conservación & 0 & 5 & 25 & 45 & 1,00 \\
\hline
\end{tabular}

Hay que indicar que el ámbito de aplicación de dicho método son las estructuras de mampostería no reforzada y de hormigón (con factores, pesos y criterios de uso diferentes), por lo que no se han podido calcular los Índices de vulnerabilidad para el caso de las estructuras de acero. No obstante, esta circunstancia no se ha considerado sustancial, debido a la escasa presencia de este tipo de estructuras en las construcciones residenciales.

La aplicación de este método permitió ajustar y verificar la clasificación contenida en la Tabla 1 respecto a las estructuras de fábrica y hormigón.

\subsection{Asignación de vulnerabilidad}

Para llevar a cabo la asignación de la vulnerabilidad a los edificios de cada localidad, se han considerado tres variables fundamentales: la edad del edificio, su altura y su localización.

\subsubsection{Edad del edificio}

Parece razonable considerar que construcciones más recientes, normalmente de pórticos de hormigón armado, son menos vulnerables que las tradicionales de muros de fábrica.

Los primeros criterios de diseño antisísmico en España surgen a raíz del terremoto de Torrevieja en 1829, a través de unos informes técnicos de construcción antisísmica que se elaboraron y en los que se aconsejaba que, en la reconstrucción de los pueblos afectados, se aumentara la anchura de las calles, se redujese la altura de las casas a un solo piso, se utilizara la maderación muy trabada entre si y se advertía de no incluir elementos exteriores decorativos como remates o cornisas.

En el siglo XX, las décadas de los años 50 y 60 se caracterizan por un vacío de normativa. Es a finales de los años 60 cuando aparece la Instrucción para el proyecto y ejecución de obras a base de hormigón armado, EH-68 (14), que constituye un hito importante, pues se cambia el modelo de cálculo utilizado hasta entonces y se amplía el control de calidad de los materiales y su ejecución. En este mismo año, la Comisión Interministerial encargada de formular las Normas sismorresistentes, elaboró la Norma Sismorresistente PGS-1-1968 (15). En 1974 se aprueba la Norma Sismorresistente PDS-1-1974 (16), así como la constitución de la Comisión Permanente de Normas Sismorresistentes. Desde este momento, las normas deberían incluir una zonificación del territorio, indicando las características de los sismos máximos que han de considerarse, unos métodos de cálculo y unas recomendaciones de uso obligado, o no, dependiendo de las zonas y de las construcciones. En 1995 se publica la Norma NCSE-94 (17), que incluía un mapa de peligrosidad sísmica de tipo probabilista, expresado en términos de aceleración sísmica básica. A partir de este año, es posible considerar que los edificios tienen un mejor diseño sismorresistente y, en consecuencia, un mejor comportamiento en caso de sismo. En 2002 dicha normativa se revisa y se publica la NCSE-O2 (1), vigente en la actualidad.

Hasta principios del siglo XX, la práctica totalidad de la edificación estaba construida mediante sistemas de muros portantes de piedra o ladrillo y es a partir de 1920 cuando comienza a construirse mediante estructuras porticadas de hormigón que se generalizan en los años 60 (9).

De la consulta de los informes técnicos de estructuras en la Comunidad Valenciana (8), se ha podido comprobar que se han localizado estructuras porticadas en un $74 \%$ de los casos. Parece claro que, a partir de los años 50, se abandonan los métodos tradicionales de construcción de muros de carga y se adopta el sistema porticado. Además, se puede estimar que sólo en la mitad de los edificios la estructura es exclusivamente de pórticos de hormigón, ya que en el resto de los casos, son estructuras mixtas y, sólo en un porcentaje inferior al $10 \%$, la estructura es exclusivamente de muros de carga.

En la actualidad, se puede afirmar que el sistema estructural predominante en las construcciones de nueva planta es el de pórticos de hormigón armado, lo que ofrece un panorama mucho más homogéneo que el correspondiente a la década de los 40 y 50 donde predominaban los muros de carga junto con el uso de estructuras metálicas y mixtas.

Para clasificar los edificios por año de construcción, se han adoptado los periodos considerados en el Censo 2001 que divide en rangos decenales el parque de edificios, a excepción del último decenio que está organizado de forma anual. 


\subsubsection{Altura del edificio}

Se puede partir de la hipótesis de que los edificios con un número de alturas superior a cinco plantas están construidos con pórticos de hormigón, ya que para alturas superiores se rebasa las resistencias admisibles de los muros de fábrica. No obstante, este factor tiene poca trascendencia en el parque de viviendas de la Comunidad Valenciana. Tras el análisis de los más de 910.00o edificios de viviendas registrados en el Censo 2001, se ha podido comprobar que el $95 \%$ de los edificios tienen alturas iguales o inferiores a cinco plantas, lo que denota una marcada horizontalidad en el parque edificado no apreciándose grandes diferencias entre las tres provincias. Estos datos coinciden con lo expuesto en el documento del IVE (8), donde se observa que el $80 \%$ de los edificios analizados en la provincia de Castellón tienen menos de cuatro alturas y el $34 \%$ son edificios de una sola planta. La provincia de Alicante presenta proporciones muy importante de edificios de cuatro y cinco alturas y la de Valencia tiene una distribución de plantas más homogénea. Por su parte, si analizamos las principales ciudades veremos cómo en Valencia capital el 50 \% de los edificios tiene alturas inferiores a cinco plantas, mientras que en Alicante y Castellón el porcentaje asciende al $75 \%$.

\subsubsection{Localización del edificio}

Se ha considerado la localización del edificio distinguiendo entre municipios en ámbito urbano o rural y poblaciones mayores o menores de 2000 habitantes. Esta agrupación se fundamenta en que los edificios ubicados en entornos rurales, utilizaban materiales, sistemas estructurales y procedimientos de ejecución de carácter local, debido a su mayor aislamiento y lejanía respecto de las áreas más industrializadas, con mayor posibilidad de acceso a las nuevas técnicas y materiales. En este sentido, se da la circunstancia de que en la provincia de Castellón, el 80 \% de sus municipios tienen una población inferior a 2000 habitantes situados en contextos rurales. Con ello la hipótesis de partida asigna una vulnerabilidad mayor a la provincia de Castellón.

Esta hipótesis viene avalada en el documento del IVE (8), donde se ha podido comprobar que, en Castellón, el $54 \%$ de los edificios tienen estructura de muros de carga. Sin embargo en Valencia y en Alicante este porcentaje queda reducido a un $5 \%$.

\subsection{Matrices de distribución de la vulnerabilidad}

Se han elaborado dos matrices de distribución de vulnerabilidad atendiendo a la localización urbana o rural de la edifica- ción, cada una de ellas organizadas por los periodos de tiempo considerados en el trabajo. Asimismo, dentro de cada periodo, se ha efectuado un estudio de detalle para estimar los porcentajes con los que distribuir los tipos estructurales considerados: muros de fábrica, sistemas mixtos, pórticos de hormigón armado sin diseño sismorresistente y pórticos de hormigón armado con diseño sismorresistente, según NCSE-94. Por último, dentro de cada tipo estructural se ha efectuado una aproximación a cuál sería la distribución de vulnerabilidad en porcentajes, según las clases consideradas A, B, C y D (Tabla 2).

Este estudio se desarrolló en varias fases. La primera consistió en elaborar una propuesta de distribución de vulnerabilidades, fundamentalmente a partir de la información contenida en el documento del IVE (8). Posteriormente fue sometida a la opinión y discusión por parte de expertos desde distintos ámbitos, tanto técnicos (ingenieros, arquitectos y aparejadores) como promotores, fabricantes y constructores que trabajaron en los años considerados dentro del sector de la construcción. Para ello se realizaron iteraciones sucesivas de un cuestionario a fin de generar convergencia de opiniones.

La Tabla 3 recoge un ejemplo para el periodo 1960-1970, en localización urbana.

Desarrollando esta tabla para cada uno de los periodos establecidos y en los dos ámbitos, rural y urbano, se obtienen las matrices para asignar clases de vulnerabilidad a los edificios de cada municipio. La Figura 2 representa los valores finales establecidos para la matriz en ámbito urbano, que ilustran la evolución cronológica de las clases de vulnerabilidad.

En la Figura 2, se puede observar cómo la mayoría de edificios construidos con anterioridad a 1940 tienen una vulnerabilidad de clase A y sólo algunos de clase B. En la década entre los años 50 y 60 la clase más representativa es la B, y a partir de 1980 la $\mathrm{C}$, considerando que prácticamente ya no se construyen edificios con vulnerabilidad A. Por último, es a partir de 1995 cuando empieza a tener un cierto peso la vulnerabilidad D.

\subsection{Mapas de vulnerabilidad sísmica}

A partir de la aplicación de las matrices de distribución de vulnerabilidad, se han obtenido los datos necesarios para realizar un conjunto de cartografías generadas mediante SIG. Los resultados indican los porcentajes y número de edificios, con una determinada vulnerabilidad, para cada municipio. En la Figura 3 podemos apreciar una muestra de dicha cartografía, cuyos resultados serán objeto de comentario más adelante.

Tabla 3. Distribución de vulnerabilidad total y por tipo estructural, para el periodo 1960-1970, en localización urbana.

\begin{tabular}{|c|c|c|c|c|c|c|}
\hline \multicolumn{3}{|c|}{ Periodo } & \multicolumn{4}{|c|}{ 1961-1970 } \\
\hline \multicolumn{3}{|c|}{ Tipología estructural } & Mural & Mixta & $\begin{array}{l}\text { Porticada hormigón armado } \\
\text { sin diseño sismorresistente }\end{array}$ & $\begin{array}{c}\text { Porticada hormigón armado } \\
\text { con diseño sismorresistente } \\
\text { y NCSE-94 }\end{array}$ \\
\hline \multicolumn{3}{|c|}{ Distribución de tipos estructurales } & o \% & $45 \%$ & $55 \%$ & o \% \\
\hline \multirow{4}{*}{$\begin{array}{l}\text { Distribución de } \\
\text { vulnerabilidad en } \\
\text { localización urbana }\end{array}$} & $\mathbf{A}$ & $14 \%$ & o \% & $25 \%$ & $5 \%$ & o \% \\
\hline & B & $48 \%$ & o \% & $75 \%$ & $25 \%$ & o \% \\
\hline & $\mathbf{C}$ & $39 \%$ & o \% & o \% & $70 \%$ & o \% \\
\hline & $\mathbf{D}$ & $\mathbf{0} \%$ & o \% & o \% & o \% & o \% \\
\hline
\end{tabular}




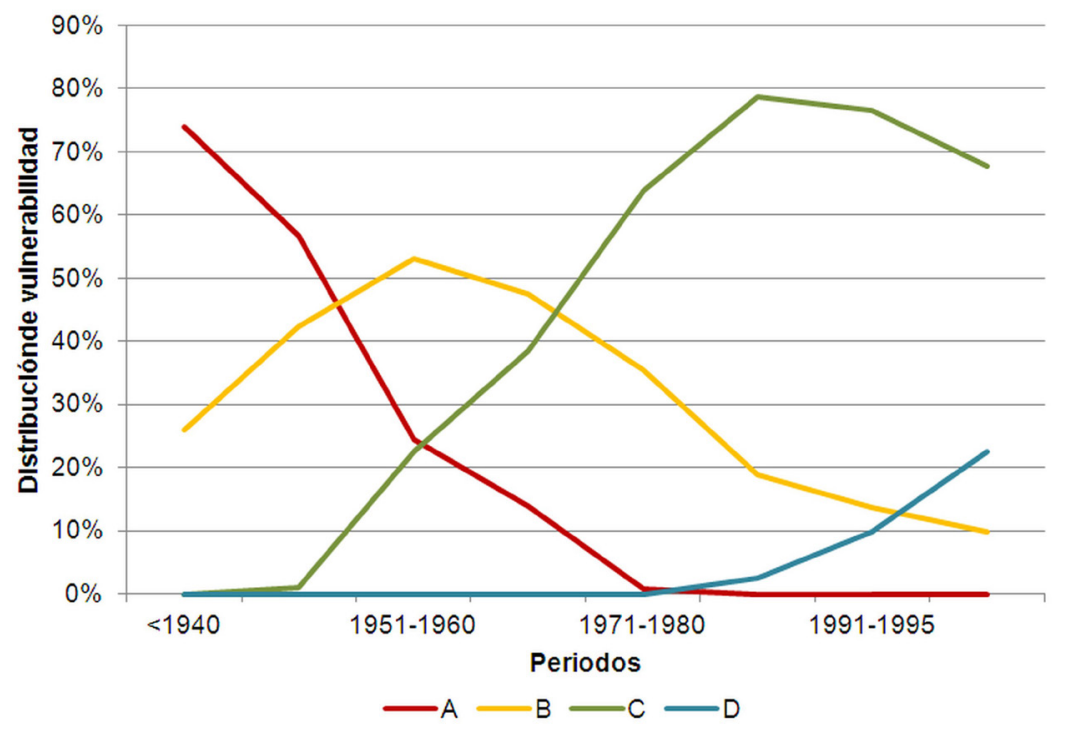

Figura 2. Vulnerabilidad en localización urbana

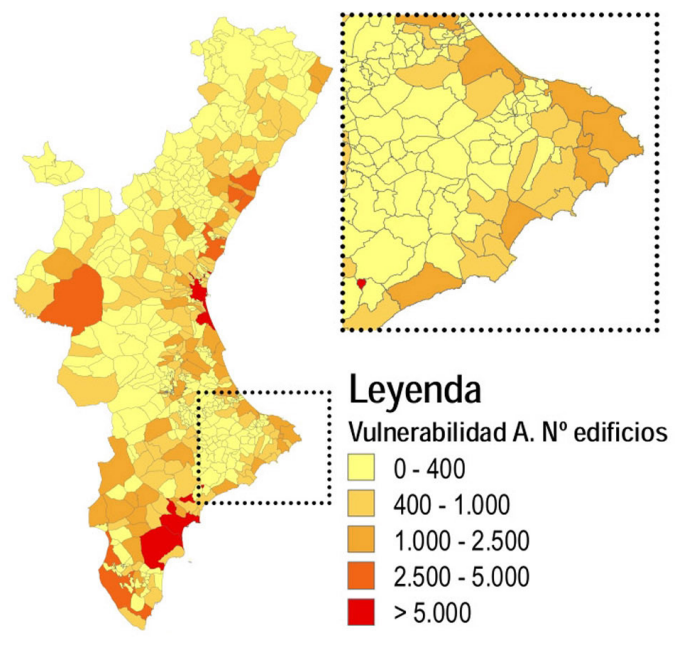

a)

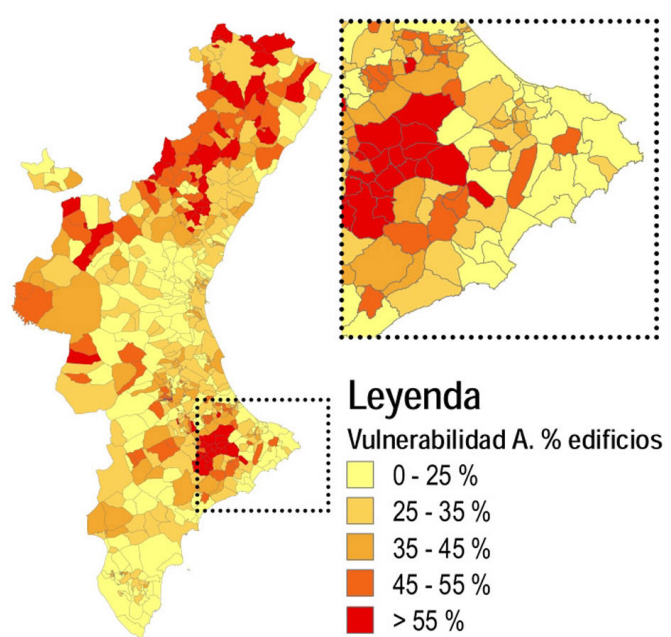

b)

Figura 3. Mapa de vulnerabilidad clase A por municipios: a) Número de edificios, b). Porcentaje de edificios.

\section{ESTIMACIÓN DEL DAÑO}

Dada la amplitud del ámbito del estudio, para cuantificar el daño se han utilizado matrices de probabilidad de daño, que constituyen una manera de expresar la probabilidad discreta de alcanzar un grado de daño, según el nivel de intensidad macrosísmica del movimiento del suelo y la clase de vulnerabilidad del edificio.

Estas matrices pueden obtenerse a partir de estudios y análisis estadísticos de los efectos y daños observados después de la ocurrencia de sismos reales o mediante el juicio de expertos. Entre otras, hay que destacar las matrices incluidas en la Applied Technology Council-13 (ATC-13), (18), y las desarrolladas por Chávez, (19). En la Comunidad Valenciana no se tienen registros sobre sismos recientes de intensidades elevadas y, en consecuencia, tampoco se tienen datos sobre los daños producidos en los edificios. Por ello, se ha recurrido a las matrices utilizadas en otras zonas con tipos estructurales y características de movimiento sísmico similares.
En este sentido, Chávez elaboró unas matrices de probabilidad de daño, basándose en los datos recogidos por el Gruppo Nazionale per la Difesa dai Terremoti (GNDT), tras inspeccionar más de 32.000 edificios que habían estado sometidos a la acción del sismo ocurrido en el año 1980 en Irpinia, al sur de Italia. Estas matrices han sido utilizadas en los estudios de riesgo sísmico desarrollados en Cataluña y en la Región de Murcia y también se han empleado para la Comunidad Valenciana.

De igual forma que se había procedido para asignar vulnerabilidad a los edificios, en este apartado se ha utilizado como base la EMS-98 (2), considerando los seis grados de daño que propone y las clases de vulnerabilidad. Sólo se han considerado las clases de la $\mathrm{A}$ hasta a la $\mathrm{D}$, al descartar los tipo $\mathrm{E}$ y $\mathrm{F}$, por considerar que apenas existen edificios en la Comunidad Valenciana con esas clases. Relacionando estos factores mediante las matrices de probabilidad de daño, se obtiene el porcentaje de daño generado por la acción de un sismo, caracterizado por una intensidad concreta, en función de la clase de vulnerabilidad del edificio. 
Los seis grados de daño con los que se ha trabajado, finalmente se reagrupan en tres, identificados como daño leve, moderado y grave, de manera que los grados de daño o y 1 quedan incluidos en el grupo de daño leve, los correspondientes a los grados 2 y 3 , en el grupo de daño medio y por último, los grados de daño 4 y 5 , en el grupo de daño grave.

El riesgo sísmico se ha representado en términos de daño, cartografiando los resultados obtenidos. La Figura 4 da muestra de ello dejando los comentarios de su contenido para más adelante.

En el estudio original se elaboró un capítulo estimando los efectos sobre la población, omitido en este artículo atendiendo a razones de extensión. No obstante, a título informativo se indica que se han utilizado dos metodologías para poder evaluar los efectos sobre la población. En concreto, la incluida en la ATC-13 (18) y la planteada por Coburn (20). Ambas ya han sido utilizadas en la elaboración de los planes de emergencia de Cataluña (21), y de Murcia (7).

\section{RESULTADOS}

\subsection{Vulnerabilidad de los edificios}

En el mapa de distribución de vulnerabilidad A representado en porcentaje (Figura 3b) se observa cómo los máximos valores alcanzados en los municipios oscilan entre un 70 y un $76 \%$, coincidiendo con términos municipales que cuentan con muy pocos edificios, entre 54 y 233 unidades, con una población que no supera los 240 habitantes y ubicados la mayor parte en la provincia de Castelló (Figura 3a). El valor medio de la vulnerabilidad A para toda la Comunidad Valenciana es del $33 \%$. Por provincias, los porcentajes para Castellón, Alicante y Valencia son del 43 \%, 32 \% y 30 \% respectivamente, lo que demuestra que la provincia de Castellón posee una vulnerabilidad de clase A superior al resto de la comunidad.

En los mapas de distribución de vulnerabilidad B y C se comprueba que estas clases de vulnerabilidades son las que predominan en la mayoría de los edificios, aunque los porcentajes para las clases A, B y C están todos alrededor del $30 \%$. La diferencia más apreciada se obtiene con la vulnerabilidad de clase $\mathrm{D}$, con un porcentaje medio del $2 \%$, siendo la clase menos frecuente en toda la región, con un porcentaje máximo de un $7 \%$ en municipios de Alicante y Valencia, disminuyendo este valor hasta el $3 \%$ en la provincia de Castellón.

Los porcentajes más altos para la vulnerabilidad $\mathrm{C}$ varían desde un $60 \%$ hasta un $76 \%$ de las edificaciones y pertenecen a municipios de las provincias de Alicante y Valencia, con población en general, por encima de los 4.000 habitantes.

\subsection{Estimación del daño en los edificios}

En el mapa de distribución de edificios con porcentaje de daño leve (Figura 4a), se observa que los valores más altos se alcanzan en los municipios de la provincia de Castellón, con un máximo próximo al $95 \%$ y con una media del $78 \%$. Por el contrario, el valor medio en Alicante es del $43 \%$ y del $55 \%$ para Valencia. Esto indica que un $58 \%$ de edificios de la Comunidad Valenciana no experimentarían daños moderados ni graves.

Consultando el mapa de distribución de edificios con porcentaje de daño grave (Figura 4b), se aprecia que el mayor valor es del $44 \%$ y se presenta en la provincia de Alicante, coincidiendo con la zona de mayor peligrosidad sísmica y con algunas de las áreas de mayor densidad de población y edificios. El valor medio en esta provincia es de aproximadamente el $13 \%$ del número de edificios, disminuyendo en las provincias de Valencia y Castellón hasta el 8 \% y el o,2 \%, respectivamente. Ello indica que en Castellón apenas se verían afectados los edificios con daños graves, aunque en el caso de porcentajes de daño moderado el valor aumenta hasta el $10 \%$, por lo que sí experimentarían daños de grado 2 y 3, según la escala EMS-98 (2). En el caso de Valencia este porcentaje es del $37 \%$ y del $44 \%$ en la provincia de Alicante, coincidiendo de nuevo con el mayor valor de porcentaje de daño moderado, llegando a máximos del $56 \%$ del número de edificios.

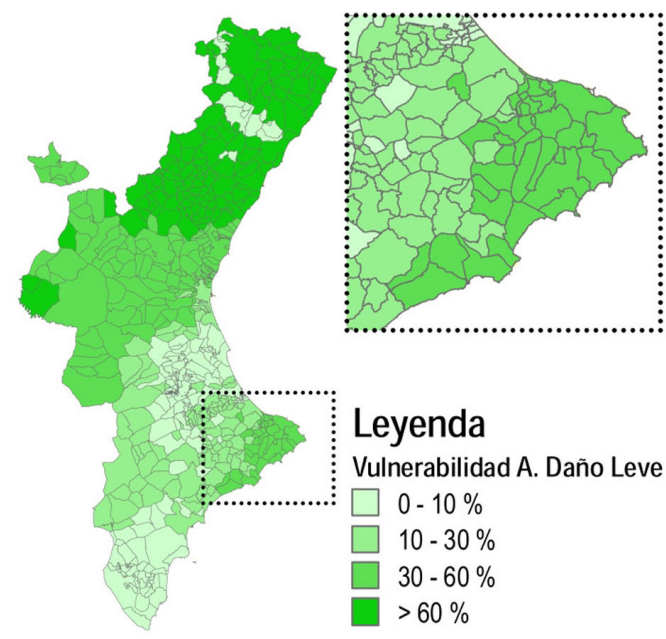

a)

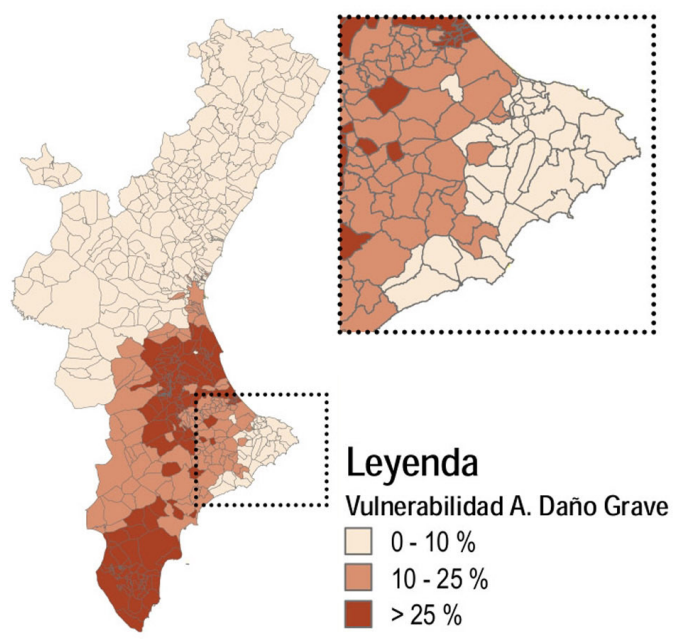

b)

Figura 4. Mapa de daño para la vulnerabilidad clase A por municipios, en porcentaje . a) Daño leve, b) Daño grave. 


\subsection{Municipios de más población e intensidad sísmica}

La Tabla 4 contiene información sobre los municipios en relación al número de habitantes e intensidad, para cada provincia.

En la Tabla 4, se observa cómo existen 4 municipios que tienen un población superior a 20.000 habitantes y, a su vez, intensidades esperadas iguales o superiores a 8,50, hecho que les confiere un riesgo especial, al concentrar mayor población y número de edificios, en áreas de notable peligrosidad. En este sentido, las Tabla 5 y 6 , muestran un resumen de la información generada para esos 4 municipios:
Según datos del Instituto Nacional de Estadística, el parque residencial de Torrevieja se construyó principalmente en las décadas de los 70 y 80, con un ritmo acelerado que produjo un relevante crecimiento en el parque de viviendas, por lo que tiene un parque de menor antigüedad que el resto de municipios y, en consecuencia, un menor porcentaje de edificios con vulnerabilidad $\mathrm{A}$, siendo la clase $\mathrm{C}$ la mayoritaria con una valor del $68 \%$. No obstante, analizando la Tabla 6, se observa como dicho municipio presenta los mayores porcentajes de daño moderado y grave, del 51,6 y 29,8, respectivamente, respecto del total de edificios. Ello hace que también alcance el mayor porcentaje de edificios inhabitables, $43 \%$. El resto de municipios presenta unos valores más similares entre sí y por debajo de los de Torrevieja.

Tabla 4. Número de municipios clasificados por intensidad máxima esperada y número de habitantes para cada provincia.

\begin{tabular}{|c|c|c|c|c|}
\hline \multirow{2}{*}{ Intensidad } & \multirow{2}{*}{$\begin{array}{c}\text { Número } \\
\text { de habitantes }\end{array}$} & \multicolumn{3}{|c|}{ Provincia } \\
\hline & & Alicante & Castellón & Valencia \\
\hline \multirow{3}{*}{$4,5-6$} & $<\mathbf{2 0 0 0}$ & o & 108 & 48 \\
\hline & $\geq \mathbf{2 0 0 0}<\mathbf{2 0 0 0 0}$ & 0 & 20 & 11 \\
\hline & $\geq \mathbf{2 0 0 0 0}$ & O & 7 & 1 \\
\hline \multirow{3}{*}{$6,5-7$} & $<\mathbf{2 0 0 0}$ & 16 & $\mathrm{O}$ & 15 \\
\hline & $\geq \mathbf{2 0 0 0}<\mathbf{2 0 0 0 0}$ & 15 & o & 36 \\
\hline & $\geq \mathbf{2 0 0 0 0}$ & 3 & $\mathrm{O}$ & 7 \\
\hline \multirow{3}{*}{$7,5-8$} & $<\mathbf{2 0 0 0}$ & 49 & $\mathrm{O}$ & 74 \\
\hline & $\geq \mathbf{2 0 0 0}<\mathbf{2 0 0 0 0}$ & 20 & $\mathrm{O}$ & 52 \\
\hline & $\geq \mathbf{2 0 0 0 0}$ & 8 & $\mathrm{o}$ & 12 \\
\hline \multirow{3}{*}{$8,5-9$} & $<\mathbf{2 0 0 0}$ & 7 & O & 6 \\
\hline & $\geq \mathbf{2 0 0 0}<\mathbf{2 0 0 0 0}$ & 16 & O & 3 \\
\hline & $\geq \mathbf{2 0 0 0 0}$ & 3 & 0 & $\mathrm{O}$ \\
\hline \multirow{3}{*}{$9,5-10$} & $<2000$ & $\mathrm{O}$ & $\mathrm{O}$ & $\mathrm{O}$ \\
\hline & $\geq \mathbf{2 0 0 0}<20000$ & 3 & $\mathrm{O}$ & $\mathrm{O}$ \\
\hline & $\geq \mathbf{2 0 0 0 0}$ & 1 & o & $\mathrm{O}$ \\
\hline
\end{tabular}

Tabla 5. Distribución de la vulnerabilidad de edificios en municipios de la Comunidad Valenciana.

\begin{tabular}{|l|c|r|r|r|r|r|r|r|r|}
\hline \multirow{2}{*}{ Municipio } & \multirow{2}{*}{$\begin{array}{c}\text { Número de } \\
\text { edificios }\end{array}$} & \multicolumn{4}{|c|}{ Número } & \multicolumn{4}{c|}{ Porcentaje } \\
\cline { 3 - 11 } & & \multicolumn{1}{c|}{ A } & \multicolumn{1}{c|}{ B } & \multicolumn{1}{c|}{ C } & \multicolumn{1}{c|}{ D } & A & B & C & D \\
\hline Crevillente & 5.969 & 1.423 & 2.076 & 2.371 & 99 & 24 & 35 & 40 & 2 \\
\hline Elche & 23.083 & 5.152 & 8.431 & 8.956 & 549 & 22 & 37 & 39 & 2 \\
\hline Orihuela & 18.518 & 3.969 & 6.448 & 7.554 & 556 & 21 & 35 & 41 & 3 \\
\hline Torrevieja & 30.514 & 847 & 7.019 & 20.638 & 2.013 & 3 & 23 & 68 & 7 \\
\hline
\end{tabular}

Tabla 6. Distribución del daño en edificios de municipios de la Comunidad Valenciana.

\begin{tabular}{|c|c|c|c|c|c|c|c|c|}
\hline \multirow{3}{*}{ Municipio } & \multicolumn{6}{|c|}{ Edificios con daño } & \multirow{2}{*}{\multicolumn{2}{|c|}{$\begin{array}{c}\text { Edificios } \\
\text { inhabitables }\end{array}$}} \\
\hline & \multicolumn{3}{|c|}{ Número } & \multicolumn{3}{|c|}{ Porcentaje } & & \\
\hline & Leve & Moderado & Grave & Leve & Moderado & Grave & Número & $\%$ \\
\hline Crevillente & 1.688 & 2.927 & 1.353 & 28,3 & 49,0 & 22,7 & 2.040 & 34 \\
\hline Elche & 6.619 & 11.354 & 5.116 & 28,7 & 49,2 & 22,2 & 7.769 & 34 \\
\hline Orihuela & 5.490 & 9.070 & 3.968 & 29,6 & 49,0 & 21,4 & 6.063 & 33 \\
\hline Torrevieja & 5.677 & 15.732 & 9.108 & 18,6 & 51,6 & 29,8 & 13.209 & 43 \\
\hline
\end{tabular}




\section{CONCLUSIONES}

La metodología que se ha seguido ya había sido aplicada en la Región de Murcia y mediante este estudio se ha constatado que es extrapolable a otras regiones, tan sólo identificando las características constructivas propias, así como la antigüedad y periodos de construcción de sus edificios. Dicha metodología, aunque basada en datos muy generales, ha permitido efectuar una clasificación del parque residencial existente en la Comunidad Valenciana y estimar los daños potenciales ocasionados por fenómenos sísmicos, a los efectos necesarios para la planificación de emergencias de Protección Civil.

Se ha comprobado la escasa información detallada sobre las características constructivas del parque edificado, así como de su estado de conservación. De ahí que se extraiga como resultado de este trabajo, el interés por promover una realización más exhaustiva y completa de las inspecciones técnicas de edificios (ITE). Esto permitiría generar una base de datos técnicos del parque construido, de gran valor para el desarrollo de futuras investigaciones. Además en los municipios con intensidades iguales o superiores a VII, sería de gran interés incorporar en dichos procedimientos, métodos de estimación de la vulnerabilidad de las construcciones. En este sentido, el método del índice de vulnerabilidad (11), aplicado en el presente estudio, se presenta como un procedimiento eficaz ya experimentado con buenos resultados en Italia.
La gestión de los datos alfanuméricos y gráficos mediante una geodatabase administrada a través de un SIG se ha demostrado como la herramienta más eficaz para este tipo de trabajos.

Los municipios son las unidades de estudio en este trabajo. Desarrollos futuros deberían adoptar unidades más pequeñas: distritos, barrios, unidad censal, etc. especialmente en grandes núcleos urbanos y con elevada concentración de población. Se deberían desarrollar estudios con más detalle, especialmente en municipios con intensidades macrosísmicas iguales o superiores a VII. También deberían desarrollarse estudios específicos en esos municipios que tengan un número de habitantes superior a 20000, dado que a mayor concentración de personas y edificios, el riesgo de daños a la población aumenta.

En el presente estudio, los mapas elaborados se refieren a un sismo hipotético en el cual se da simultáneamente la intensidad máxima esperada en cada municipio. Obviamente esta situación es irreal, pues los efectos de un sismo se atenúan en función de la distancia a su epicentro o foco y en consecuencia, la intensidad no es constante. Por ello se propone en futuros desarrollos, la elaboración de mapas de isosistas que muestren una posible distribución de intensidades macrosísmicas que permita establecer unos escenarios de daño más realistas y, en consecuencia, de mayor utilidad y efectividad en la prevención del riesgo sísmico.

\section{REFERENCIAS}

(1) Ministerio de Fomento. (2002, 11 de octubre). Real Decreto 997/2002, de 27 de septiembre de 2002, por el que se aprueba la NCSE-02 Norma de Construcción Sismorresistente: parte General y de Edificación. Boletín Oficial del Estado, $n^{0}$ 244, pp. 35898-35967. España.

(2) Grünthal, G. (1998). European Macroseismic Scale 1998, vol. 7. Luxembourg: Cahiers du Centre Européen de Géodynamique et de Séismologie.

(3) Giner-Caturla, J. J. (2011). Estudio sobre Peligrosidad Sísmica de la Comunitat Valenciana. Alicante: Universidad de Alicante y Consorcio de Bomberos de la Diputación de Alicante.

(4) Consellería de Gobernación y Justicia. (2011, 3 de mayo). Decreto 44/2011, de 29 de abril, del Consell, Diario Oficial de la Comunidad Valenciana, por el que se aprueba El Plan Especial frente al Riesgo Sísmico en la Comunitat Valenciana. Diario oficial de la Comunidad Valenciana, no 6512.

(5) Sandi, H. (1983). Earthquake risk and earthquake preparedness: some qualitative aspects and quantification possibility. En Proceedings of the Seminar on Earthquake Preparedness (pp. 79-93). UNDP/UNESCO/UNDRO Project for Earthquake Risk Reduction in the Balkan Region.

(6) Perepérez, B. (2014). La peligrosidad sísmica y el factor de riesgo. Informes de la Construcción, 66(534): e018, doi: http://dx.doi.org/10.3989/ic.13.018.

(7) Consejería de Presidencia DG de Protección Civil. (2006). RISMUR. Plan Especial de Protección Civil ante el Riesgo Sísmico en la Región de Murcia. Región de Murcia. Homologado por la Comisión Nacional de Protección Civil el 29 de julio de 2006.

(8) Serrano, B., De Mazarredo, F.C., Osorio, A., Palencia, J.J., García-Prieto, S. (2008). Experiencia en Inspección de estructuras en edificios. Comunitat Valenciana 1991-20o8 (pp. 43-53). Valencia: Generalitat Valenciana e Instituto Valenciano de la Edificación.

(9) Temes-Cordovez, R. (2007). El tapiz de Penélope. Transformaciones residenciales sobre tejidos sin valor patrimonial (Tesis doctoral inédita). Valencia: Escuela Técnica Superior de Arquitectura - Universidad Politécnica de Valencia, doi: http://hdl.handle.net/10251/2906.

(10) Marco-Serrano, E. (2012). Análisis de la normativa de hormigón armado en España y la influencia de los investigadores españoles desde 1939 a 1973. Aplicación de la técnica constructiva en la ciudad de Valencia (Tesis doctoral inédita). Valencia: Escuela Técnica Superior de Arquitectura - Universidad Politécnica de Valencia, doi: http://hdl.handle. net/10251/17124.

(11) Benedetti, D., Petrini, V. (1984). Sulla vulnerabilitá sismica di edifici in muratura. Proposte di un metodo di valutazione. L'industria delle Costruzioni, 149: 66-78.

(12) Yépez, F. (1996). Metodología para la evaluación de la vulnerabilidad y riesgo sísmico de estructuras aplicando técnicas de simulación (Tesis doctoral). Barcelona (Spain): Dpto. Ingeniería del Terreno, Cartográfica y Geofísica - Universidad Politécnica de Cataluña. 
(13) Mena, U. (2002). Evaluación del Riesgo Sísmico en Zonas Urbanas (Tesis doctoral). Barcelona: Dpto. Ingeniería del Terreno, Cartográfica y Geofísica - Universidad Politécnica de Cataluña.

(14) Presidencia del Gobierno. (1968, 3 de diciembre). Decreto 2987/1968, de 20 de septiembre de 1968, por el que se aprueba la EH-68. Instrucción para el proyecto y la ejecución de obras de hormigón en masa o armado. Boletín Oficial del Estado, nº 290, pp. 17257-17291. España.

(15) Presidencia del Gobierno. (1969, 4 de febrero). Decreto 106/1969, de 16 de enero de 1969, por el que se aprueba la PGS1-1968 Norma Sismorresistente: parte A. Boletín Oficial del Estado, n⿳0 30, pp. 1658-1675.

(16) Ministerio de Planificación del Desarrollo. (1974, 21 de noviembre). Decreto 3209/1974, de 30 de agosto de 1974, por el que se aprueba la PDS-1-1974 Norma Sismorresistente: parte A. Boletín Oficial del Estado, n 279, pp. 23585-23601. España.

(17) Ministerio de Obras Públicas, Transporte y Medio Ambiente. (1995, 8 de febrero). Real Decreto 2543/1994, de 29 de diciembre de 1994, por el que se aprueba la NCSE-94 Norma de Construcción Sismorresistente: parte General y de Edificación. Boletín Oficial del Estado, no 33 , pp. 3935-3980. España.

(18) FEMA. (1985). ATC-13. Applied Technology Council. Earthquake damage evaluation data for California. Redwood City, CA: Federal Emergency Management Agency.

(19) Chávez, J. (1998). Evaluación de la vulnerabilidad y el riesgo sísmico a escala regional: aplicación a Cataluña (Tesis doctoral inédita). Barcelona: Universidad Politécnica de Cataluña.

(20) Coburn, A., Spence, R. (1992). Earthquake protection. New York: John Wiley \& Sons.

(21) Departament de Justícia i Interior. (2003, 26 de junio). JUI/1915/2003, de 20 de maig de 2003, por el que se aprueba SISMICAT. Pla Especial d'Emergències Sísmiques a Catalunya. Diari Oficial de la Generalitat de Catalunya, $\mathrm{n}^{\mathrm{0}} 3912$. 